\title{
Impossible Differential Cryptanalysis of CLEFIA
}

\author{
Yukiyasu Tsunoo ${ }^{1}$, Etsuko Tsujihara $^{2}$, Maki Shigeri $^{3}$, Teruo Saito $^{3}$, \\ Tomoyasu Suzaki ${ }^{1}$, and Hiroyasu Kubo ${ }^{3}$
}

${ }^{1}$ NEC Corporation, 1753, Shimonumabe, Nakahara, Kawasaki 211-8666, Japan

\{tsunoo@BL, t-suzaki@cb $\} \cdot j p . n e c . c o m$

${ }^{2}$ Y.D.K.Co., Ltd., 1288, Oshitate, Inagi-Shi, Tokyo 206-0811, Japan

etsuko-t@ghn.ydkinc.co.jp

${ }^{3}$ NEC Software Hokuriku, Ltd., 1,Anyoji, Hakusan, Ishikawa 920-2141, Japan

\{m-shigeri@pb,t-saito@qh, h-kubo@ps\}.jp.nec.com

\begin{abstract}
This paper reports impossible differential cryptanalysis on the 128-bit block cipher CLEFIA that was proposed in 2007, including new 9-round impossible differentials for CLEFIA, and the result of an impossible differential attack using them. For the case of a 128-bit key, it is possible to apply the impossible differential attack to CLEFIA reduced to 12 rounds. The number of chosen plaintexts required is $2^{118.9}$ and the time complexity is $2^{119}$. For key lengths of 192 bits and 256 bits, it is possible to apply impossible differential attacks to 13-round and 14round CLEFIA. The respective numbers of chosen plaintexts required are $2^{119.8}$ and $2^{120.3}$ and the respective time complexities are $2^{146}$ and $2^{212}$. These impossible differential attacks are the strongest method for attacking reduced-round CLEFIA.
\end{abstract}

Keywords: block cipher, CLEFIA, diffusion switching mechanism, generalized Feistel structure, impossible differential cryptanalysis.

\section{Introduction}

Differential attacks 2 and linear attacks 3 are the most common methods of attack applied to block ciphers. Guaranteeing security against differential attacks and linear attacks is an important problem in the design of block ciphers. One known method of evaluating security against such attacks uses the minimum number of active S-boxes. Shirai et al. proposed in 2004 the diffusion switching mechanism (DSM), a method of designing a Feistel structure block cipher that can guarantee a large minimum number of active S-boxes 4|5. In 2007, CLEFIA, a 128-bit block cipher designed using DSM, was proposed [6. The designers of CLEFIA adopted a four-branch generalized Feistel structure to achieve both a small implementation size and high speed. The generalized Feistel structure tends to require more rounds to guarantee security than does an ordinary Feistel structure, but CLEFIA can guarantee resistance to differential attacks and linear attacks with a small number of rounds because of the use of DSM.

The impossible differential attack [1] is a method that was first applied against Skipjack to reject wrong key candidates by using input difference and output 
difference pairs whose probabilities are zero (impossible differentials). Impossible differentials that are dependent on the basic structure of the data processing part are often used, and this method is a particular threat to the generalized Feistel structure. Since CLEFIA is a generalized Feistel structure, the impossible differential attack is an effective attack against CLEFIA. According to the designers, an evaluation of CLEFIA with respect to an impossible differential attack 6 6] shows that there are 9-round impossible differentials in CLEFIA, and for a 128-bit key, a 10-round impossible differential attack is possible. For key lengths of 192 bits and 256 bits, 11-round and 12-round impossible differential attacks are possible.

In this paper, we show that there are previously unknown 9-round impossible differentials in CLEFIA and report the result of impossible differential attacks using those impossible differentials. These impossible differentials exist in structures that are designed using DSM. In the impossible differential attacks on CLEFIA described in this paper, 12-round CLEFIA can be broken for a 128-bit key. For key lengths of 192 bits and 256 bits, impossible differential attacks are respectively possible for 13-round and 14-round CLEFIA.

There have been no reports on the cryptanalysis of CLEFIA other than the evaluation by the designers. Accordingly, the strong attack method for CLEFIA up to now is the differential attack and linear attack described in the designers' evaluation, which shows the possibility of 12-round, 13-round, and 14-round attack for the respective key lengths of 128 bits, 192 bits, and 256 bits. Nevertheless, these results are values for guaranteeing security with respect to differential attacks or linear attacks; the numbers of rounds for establishing actual differential attacks or linear attacks are probably smaller. Accordingly, the impossible differential attacks described in this paper are the result for the most number of rounds as an actual attack method on CLEFIA.

In this paper, we describe the CLEFIA structure in Sect. 2, explain the newly discovered impossible differentials and present attack procedures against CLEFIA using those differentials in Sect. 3. Section 4 concludes this paper.

\section{Description of CLEFIA}

\subsection{Notation}

We use the following notation in this paper.

$\begin{array}{ll}a_{(b)} & b \text { is the bit length of } a \\ & \text { If the bit length of } a \text { is known, }(b) \text { is omitted. } \\ & \text { The concatenation of } a \text { and } b \\ {[a, b]} & \text { The vector representation of } a \mid b \\ { }^{t} a & \text { Transposition of vector } a \text { or matrix } a \\ {\left[x^{\{i, 0\}}, x^{\{i, 1\}}, x^{\{i, 2\}}, x^{\{i, 3\}}\right]} & i \text {-round output data, } x^{\{i, j\}} \in\{0,1\}^{32} \\ & \text { The plaintext is }\left[x^{\{0,0\}}, x^{\{0,1\}}, x^{\{0,2\}}, x^{\{0,3\}}\right] . \\ {\left[C^{\{i, 0\}}, C^{\{i, 1\}}, C^{\{i, 2\}}, C^{\{i, 3\}}\right]} & \text { The } i \text {-round CLEFIA ciphertext }\end{array}$




$\begin{array}{ll}a \oplus b & \begin{array}{l}\text { Bit-wise exclusive OR of } a \text { and } b \\ \left.\text { (addition over } \operatorname{GF}\left(2^{n}\right)\right)\end{array} \\ \Delta a & \text { Difference for } a\left(\text { difference over } \operatorname{GF}\left(2^{n}\right)\right) \\ w_{b}(a) & \text { For an } 8 n \text {-bit string } \\ & a=a_{0(8)}\left|a_{1(8)}\right| \ldots \mid a_{n-1(8)}, \\ & w_{b}(a) \text { denotes the number of non-zero } a_{i} \mathrm{~s} . \\ & \text { Branch number for function } P \\ B(P) & B(P)=\min _{a \neq 0}\left\{w_{b}(a)+w_{b}(P(a))\right\}\end{array}$

\section{$2.2 \quad$ Structure}

In this section, we explain only the data processing part of CLEFIA.

CLEFIA is a block cipher that has a block length of 128 bits and key lengths of 128,192 , and 256 bits.

The data processing part is a four-branch generalized Feistel structure with two parallel $\mathrm{F}$ functions $\left(F_{0}, F_{1}\right)$ per round. The number of respective rounds $r$ for 128-bit, 192-bit and 256-bit keys are 18, 22 and 26. The encryption function $E N C_{r}$ generates 128-bit ciphertext from 128-bit plaintext, $2 r$ 32-bit round keys $\left(R K_{0(32)}, \ldots, R K_{2 r-1(32)}\right)$, and four 32 -bit whitening keys $\left(W K_{0}, \ldots, W K_{3}\right)$. The structure of the encryption function $E N C_{r}$ is shown in Fig. 1 $E N C_{r}$ is defined as follows.

$E N C_{r}$ :

Step 1. $T_{0}\left|T_{1}\right| T_{2}\left|T_{3} \leftarrow x^{\{0,0\}}\right|\left(x^{\{0,1\}} \oplus W K_{0}\right)\left|x^{\{0,2\}}\right|\left(x^{\{0,3\}} \oplus W K_{1}\right)$

Step 2. For $i=0$ to $r-1$ do the following:

Step 2.1. $T_{1} \leftarrow T_{1} \oplus F_{0}\left(R K_{2 i}, T_{0}\right), T_{3} \leftarrow T_{3} \oplus F_{1}\left(R K_{2 i+1}, T_{2}\right)$

Step 2.2. $T_{0}\left|T_{1}\right| T_{2}\left|T_{3} \leftarrow T_{1}\right| T_{2}\left|T_{3}\right| T_{0}$

Step 3. $C^{\{r, 0\}}\left|C^{\{r, 1\}}\right| C^{\{r, 2\}}\left|C^{\{r, 3\}} \leftarrow T_{3}\right|\left(T_{0} \oplus W K_{2}\right)\left|T_{1}\right|\left(T_{2} \oplus W K_{3}\right)$

The two $\mathrm{F}$ functions, $F_{0}$ and $F_{1}$, have 32-bit data $x$ and 32-bit key $R K$ as input; they output the 32 -bit data $y$. $F_{0}$ is defined as follows.

$F_{0}$ :

Step 1. $T \leftarrow R K \oplus x$

Step 2. Let $T=T_{0(8)}\left|T_{1(8)}\right| T_{2(8)} \mid T_{3(8)}$

$$
T_{0} \leftarrow S_{0}\left(T_{0}\right), T_{1} \leftarrow S_{1}\left(T_{1}\right), T_{2} \leftarrow S_{0}\left(T_{2}\right), T_{3} \leftarrow S_{1}\left(T_{3}\right)
$$

Step 3. Let $y=y_{0(8)}\left|y_{1(8)}\right| y_{2(8)} \mid y_{3(8)}$ ${ }^{t}\left[y_{0}, y_{1}, y_{2}, y_{3}\right]=M_{0}{ }^{t}\left[T_{0}, T_{1}, T_{2}, T_{3}\right]$

$F_{1}$ is defined by replacing the terms in $F_{0}$ as follows: $S_{0}$ is replaced with $S_{1}, S_{1}$ with $S_{0}$, and $M_{0}$ with $M_{1}$. The structures of $F_{0}$ and $F_{1}$ are shown in Fig. 2

$S_{0}$ and $S_{1}$ are non-linear 8-bit S-boxes.

The two matrices $M_{0}$ and $M_{1}$ are defined as

$$
M_{0}=\left(\begin{array}{ccccc}
\text { 0x01 } & \text { 0x02 } & \text { 0x04 } & \text { 0x06 } \\
\text { 0x02 } & \text { 0x01 } & \text { 0x06 } & \text { 0x04 } \\
\text { 0x04 } & \text { 0x06 } & \text { 0x01 } & \text { 0x02 } \\
\text { 0x06 } & \text { 0x04 } & \text { 0x02 } & \text { 0x01 }
\end{array}\right), M_{1}=\left(\begin{array}{ccccc}
\text { 0x01 } & \text { 0x08 } & \text { 0x02 } & \text { 0x0a } \\
\text { 0x08 } & \text { 0x01 } & \text { 0x0a } & \text { 0x02 } \\
\text { 0x02 } & \text { 0x0 } & \text { 0x01 } & \text { 0x08 } \\
\text { 0x0a } & \text { 0x02 } & \text { 0x08 } & \text { 0x01 }
\end{array}\right) .
$$




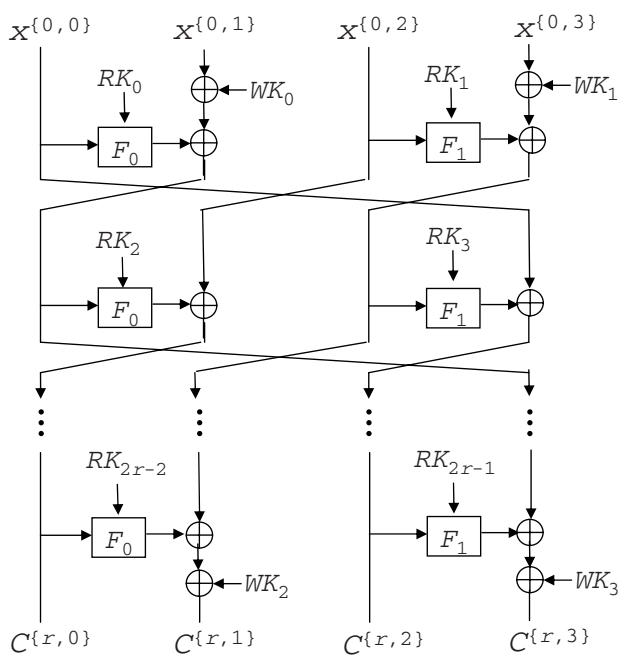

Fig. 1. Encryption function $E N C_{r}$
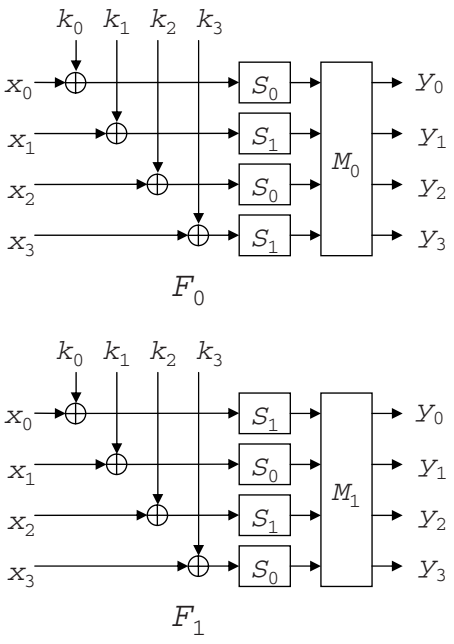

Fig. 2. Functions $F_{0}$ and $F_{1}$

The multiplications between these matrices and vectors are performed in $G F\left(2^{8}\right)$ defined by the primitive polynomial $z^{8}+z^{4}+z^{3}+z^{2}+1$.

$M_{0}$ and $M_{1}$ satisfy

$$
B\left(M_{0}\right)=B\left(M_{1}\right)=5, B\left(M_{0} \mid M_{1}\right)=B\left({ }^{t} M_{0}^{-1} \mid{ }^{t} M_{1}^{-1}\right)=5 .
$$

\section{Impossible Differential Attacks on CLEFIA}

In this section, we present the new 9-round impossible differentials in Sect. 3.1. and explain the procedure for using those impossible differentials to attack CLEFIA in Sect. 3.2 and subsequent sections.

\subsection{Nine-Round Impossible Differentials of CLEFIA}

The following two new 9-round impossible differentials are found in CLEFIA,

$$
\begin{aligned}
& {\left[0_{(32)}, 0_{(32)}, 0_{(32)}, \alpha_{\text {in }(32)}\right] \operatorname{tar}_{9 r}\left[0_{(32)}, 0_{(32)}, 0_{(32)}, \alpha_{\text {out }(32)}\right]} \\
& {\left[0_{(32)}, \alpha_{\text {in }(32)}, 0_{(32)}, 0_{(32)}\right] t_{9 r}\left[0_{(32)}, \alpha_{\text {out }(32)}, 0_{(32)}, 0_{(32)}\right]}
\end{aligned}
$$

where $\alpha_{\text {in }}$ and $\alpha_{\text {out }}$ are the differences shown in Table 1 . The $X_{(8)}$ and $Y_{(8)}$ in $\alpha_{\text {in }}$ and $\alpha_{\text {out }}$ are arbitrary non-zero values. These impossible differentials are entirely different from the impossible differentials found by the designers. The first impossible differential is represented in Fig. 3.

Here, we prove that where $\alpha_{i n}=\left[0_{(8)}, 0_{(8)}, 0_{(8)}, X_{(8)}\right]$, and $\alpha_{\text {out }}=\left[Y_{(8)}, 0_{(8)}\right.$, $\left.0_{(8)}, 0_{(8)}\right]$, the probability of $\left[0_{(32)}, 0_{(32)}, 0_{(32)}, \alpha_{i n}\right]$ occurring nine rounds after $\left[0_{(32)}, 0_{(32)}, 0_{(32)}, \alpha_{\text {out }}\right]$ is zero, which is to say that $\left[0,0,0, \alpha_{\text {in }}\right] \operatorname{tagr}_{9 r}\left[0,0,0, \alpha_{\text {out }}\right]$ is an impossible differential. Other impossible differentials can be proven in the same way. 
Table 1. Differential values for $\alpha_{\text {in }}$ and $\alpha_{\text {out }}$

\begin{tabular}{|c|c|}
\hline$\alpha_{\text {in }}$ & $\alpha_{\text {out }}$ \\
\hline$\left[0_{(8)}, 0_{(8)}, 0_{(8)}, X_{(8)}\right]$ & {$\left[0_{(8)}, 0_{(8)}, Y_{(8)}, 0_{(8)}\right],\left[0_{(8)}, Y_{(8)}, 0_{(8)}, 0_{(8)}\right],\left[Y_{(8)}, 0_{(8)}, 0_{(8)}, 0_{(8)}\right]$} \\
\hline$\left[0_{(8)}, 0_{(8)}, X_{(8)}, 0_{(8)}\right]$ & {$\left[0_{(8)}, 0_{(8)}, 0_{(8)}, Y_{(8)}\right],\left[0_{(8)}, Y_{(8)}, 0_{(8)}, 0_{(8)}\right],\left[Y_{(8)}, 0_{(8)}, 0_{(8)}, 0_{(8)}\right]$} \\
\hline$\left[0_{(8)}, X_{(8)}, 0_{(8)}, 0_{(8)}\right]$ & {$\left[0_{(8)}, 0_{(8)}, 0_{(8)}, Y_{(8)}\right],\left[0_{(8)}, 0_{(8)}, Y_{(8)}, 0_{(8)}\right],\left[Y_{(8)}, 0_{(8)}, 0_{(8)}, 0_{(8)}\right]$} \\
\hline$\left[X_{(8)}, 0_{(8)}, 0_{(8)}, 0_{(8)}\right]$ & {$\left[0_{(8)}, 0_{(8)}, 0_{(8)}, Y_{(8)}\right],\left[0_{(8)}, 0_{(8)}, Y_{(8)}, 0_{(8)}\right],\left[0_{(8)}, Y_{(8)}, 0_{(8)}, 0_{(8)}\right]$} \\
\hline
\end{tabular}

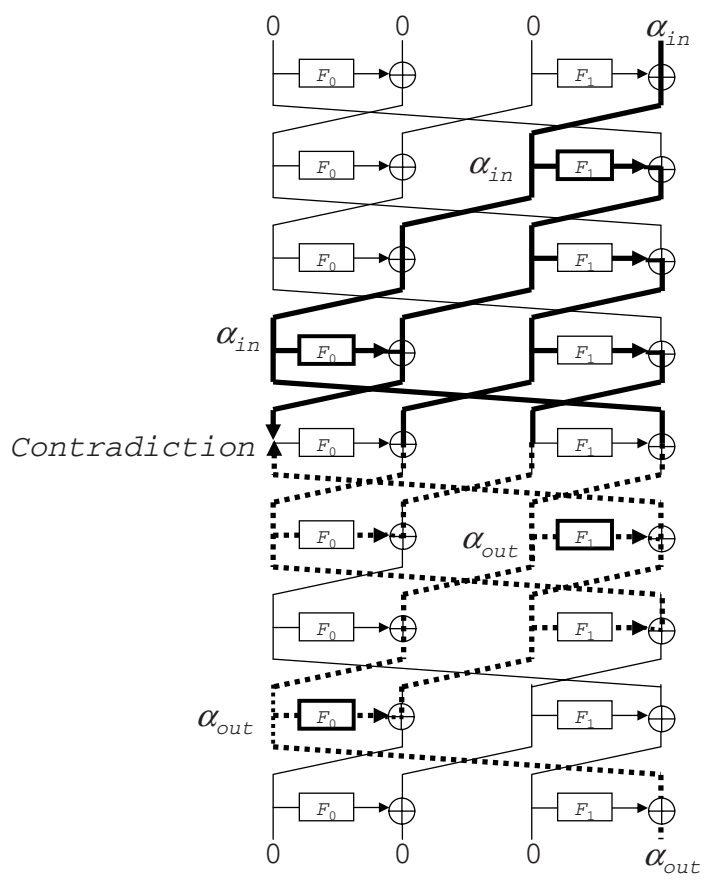

Fig. 3. Nine-round impossible differential

Proof. Assume that the input difference $\Delta x^{\{4,0\}}$ of the fifth-round $F_{0}$ function for when the input difference is $\left[0_{(32)}, 0_{(32)}, 0_{(32)},\left[0_{(8)}, 0_{(8)}, 0_{(8)}, X_{(8)}\right]\right]$ and the input difference $\Delta x^{\prime\{4,0\}}$ of the fifth-round $F_{0}$ function for when the output difference is $\left[0_{(32)}, 0_{(32)}, 0_{(32)},\left[Y_{(8)}, 0_{(8)}, 0_{(8)}, 0_{(8)}\right]\right]$ are the same.

$$
\Delta x^{\{4,0\}}=\Delta x^{\prime\{4,0\}} .
$$

The $\Delta x^{\{4,0\}}$ can be expressed using the fourth-round matrix $M_{0}$ and secondround matrix $M_{1}$ as

$$
\begin{aligned}
\Delta x^{\{4,0\}} & =M_{0}{ }^{t}\left[0,0,0, X^{\prime}\right] \oplus M_{1}{ }^{t}\left[0,0,0, X^{\prime \prime}\right] \\
& =\left(M_{0} \mid M_{1}\right)^{t}\left[0,0,0, X^{\prime}, 0,0,0, X^{\prime \prime}\right],
\end{aligned}
$$


where $X^{\prime}$ is the output difference for when the $S_{1}$ input difference is $X$, and $X^{\prime \prime}$ is the output difference for when the $S_{0}$ input difference is $X$; both are non-zero values.

Also, the $\Delta x^{\prime\{4,0\}}$ can be expressed using the 8th-round matrix $M_{0}$ and the 6th-round matrix $M_{1}$ as

$$
\begin{aligned}
\Delta x^{\prime\{4,0\}} & =M_{0}{ }^{t}\left[Y^{\prime}, 0,0,0\right] \oplus M_{1}{ }^{t}\left[Y^{\prime \prime}, 0,0,0\right] \\
& =\left(M_{0} \mid M_{1}\right)^{t}\left[Y^{\prime}, 0,0,0, Y^{\prime \prime}, 0,0,0\right],
\end{aligned}
$$

where $Y^{\prime}$ is the output difference for when the $S_{0}$ input difference is $Y$ and $Y^{\prime \prime}$ is the output difference for when the $S_{1}$ input difference is $Y$; both are non-zero values.

From (1), (2) and (3), we obtain

$$
\left(M_{0} \mid M_{1}\right)^{t}\left[Y^{\prime}, 0,0, X^{\prime}, Y^{\prime \prime}, 0,0, X^{\prime \prime}\right]={ }^{t}[0,0,0,0]
$$

because

$$
\begin{aligned}
& \Delta x^{\{4,0\}} \oplus \Delta^{\prime} x^{\{4,0\}} \\
= & \left(M_{0} \mid M_{1}\right)^{t}\left[0,0,0, X^{\prime}, 0,0,0, X^{\prime \prime}\right] \oplus\left(M_{0} \mid M_{1}\right)^{t}\left[Y^{\prime}, 0,0,0, Y^{\prime \prime}, 0,0,0\right] \\
= & \left(M_{0} \mid M_{1}\right)^{t}\left(\left[0,0,0, X^{\prime}, 0,0,0, X^{\prime \prime}\right] \oplus\left[Y^{\prime}, 0,0,0, Y^{\prime \prime}, 0,0,0\right]\right) \\
= & \left(M_{0} \mid M_{1}\right)^{t}\left[Y^{\prime}, 0,0, X^{\prime}, Y^{\prime \prime}, 0,0, X^{\prime \prime}\right] .
\end{aligned}
$$

From the CLEFIA specifications, the branch number of the concatenation matrix $M_{0} \mid M_{1}$ is 5. Therefore

$$
w_{b}\left(\left[Y^{\prime}, 0,0, X^{\prime}, Y^{\prime \prime}, 0,0, X^{\prime \prime}\right]\right)+w_{b}\left(\left(M_{0} \mid M_{1}\right)^{t}\left[Y^{\prime}, 0,0, X^{\prime}, Y^{\prime \prime}, 0,0, X^{\prime \prime}\right]\right) \geq 5 .
$$

From $w_{b}\left(\left[Y^{\prime}, 0,0, X^{\prime}, Y^{\prime \prime}, 0,0, X^{\prime \prime}\right]\right)=4$, for the left side of (4),

$$
w_{b}\left(\left(M_{0} \mid M_{1}\right)^{t}\left[Y^{\prime}, 0,0, X^{\prime}, Y^{\prime \prime}, 0,0, X^{\prime \prime}\right]\right) \geq 1 .
$$

Furthermore, for the right side of (4),

$$
w_{b}([0,0,0,0])=0 .
$$

Equations (5) and (6) contradict (44).

Accordingly, $\Delta x^{\{4,0\}}$ and $\Delta x^{\prime\{4,0\}}$ cannot be equal and $[0,0,0,[0,0,0, X]] \nrightarrow_{9 r}$ $[0,0,0,[Y, 0,0,0]]$ is thus an impossible differential.

\subsection{Key Recovery Attack on 11-Round CLEFIA}

In this section, we explain an impossible differential attack on 11-round CLEFIA using the 9-round impossible differentials presented in Sect.3.1 as preparation for an impossible differential attack on 12-round CLEFIA which we show in Sect. 3.3. For simplicity of explanation in the next section, we regard the first-round output to be plaintext and present the attack procedure for the 11 rounds from the second round to the 12 th round. Of the 9-round impossible differentials shown in Sect. 3.1. we describe the case for the input difference of $[0,0,0,[0,0,0, X]]$ and the output difference of $[0,0,0,[Y, 0,0,0]]$ as shown in Fig. 4. It is possible to recover $R K_{22}, R K_{23}$, and the most significant byte of $W K_{2} \oplus R K_{21}$, which we represent as $R K_{21,0(8)}^{\prime}$. 


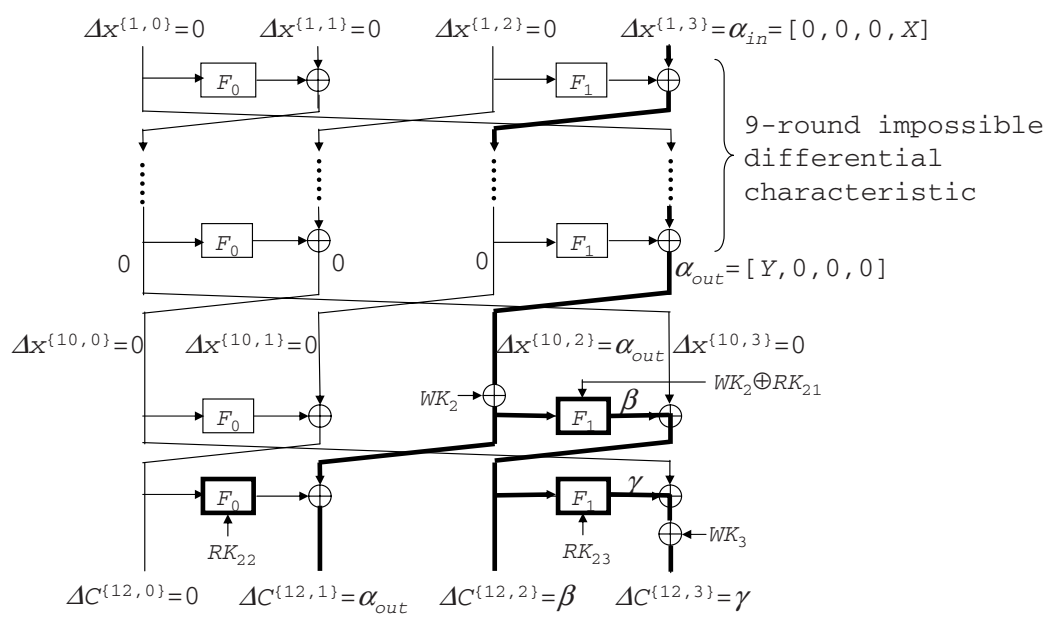

Fig. 4. Impossible differential attack on 11-round CLEFIA

Movement of Whitening Key $\boldsymbol{W} \boldsymbol{K}_{\mathbf{2}}$. Move the whitening key $W K_{2}$, and place it at the bit-wise exclusive OR with the 10th-round output $x^{\{10,2\}}$ and bitwise exclusive $\mathrm{OR}$ with $R K_{21}$. This movement is an equivalent transformation.

Key Recovery. Of the ciphertext pairs that correspond to the plaintext pairs for which the difference is $[0,0,0,[0,0,0, X]]$, choose those for which the ciphertext difference is $\left[0,[Y, 0,0,0], \beta_{(32)}, \gamma_{(32)}\right]$. Here, $\beta$ represents the 255 values that can be obtained as the output difference when the input difference for $M_{1}$ is $[Y, 0,0,0] ; \gamma$ is a non-zero value. The probability of obtaining such ciphertext pairs is $1 / 2^{32} \cdot 255 / 2^{32} \cdot 255 / 2^{32} \cdot\left(2^{32}-1\right) / 2^{32} \approx 2^{-80}$.

For the chosen ciphertext pair, all of the keys that are obtained by differential tabld look-up indexed on the input differences and the output differences of the 11th-round $F_{1}$ and the 12 th-round $F_{1}$ as the key are wrong keys. Those keys are marked as wrong keys in a key table for distinguishing whether $R K_{21,0}^{\prime}\left|R K_{22}\right| R K_{23}$ candidates 2 are correct keys or wrong keys. This method is generally used with the objective of finding the correct key by differential attacks; in impossible differential attacks, it can be used to find wrong keys without exhaustive search. The probability of a candidate for $R K_{21,0}^{\prime}\left|R K_{22}\right| R K_{23}$ being a wrong key as the result of using two $F_{1}$ differential tables is $2^{-40}$ from the average $2^{-8}$ probability for the 11 th-round $F_{1}$ and the average $2^{-32}$ probability for the 12th-round $F_{1}$. Accordingly, the number of ciphertext pairs required to

$\overline{{ }^{1} \text { A table that }}$ records the input value pairs for which occur the input-output differences for each of the input differences and output differences.

2 To calculate the input value of the 11 th-round $F_{1}$, it is necessary to try all of $R K_{22}$. It is therefore useful to have the $R K_{21,0}^{\prime} \mid R K_{23}$ key table when guessing $R K_{22}$, but we chose to add $R K_{22}$ to the key table as well to simplify the explanation of the 12 -round attack in Sect. 3.3 . 
narrow the candidates down to a single 72-bit correct key $R K_{21,0}^{\prime}\left|R K_{22}\right| R K_{23}$, $N$, is about $2^{45.7}$, from

$$
2^{72}\left(1-2^{-40}\right)^{N}=1 \text {. }
$$

From the above facts, $2^{45.7} / 2^{-80}=2^{125.7}$ plaintext pairs are required for attack. If we choose two different plaintexts from a set of $2^{8}$ plaintexts (referred to simply as 'structure' below) for which the first three words and the first three bytes of the fourth word of the plaintext are fixed, we can make ${ }_{2}{ }^{8} C_{2} \approx 2^{14.9}$ pairs for which the difference is $[0,0,0,[0,0,0, X]]$. In other words, it is possible to obtain the number of ciphertext pairs that are required for the attack by choosing $2^{110.8}\left(=2^{125.7-14.9}\right)$ structures. In that case, the number of plaintexts is $2^{110.8} \cdot 2^{8}=2^{118.8}$.

The time complexity for attack is as follows.

1. For obtaining the ciphertexts : $2^{119}$ encryptions

2. For reducing the key candidates : $2^{46} \cdot 2^{32}=2^{78}$ F-function computations $<2^{73}$ encryptions

(In detail, $2^{45.7}$ ciphertext pairs $\cdot 2^{32} R K_{22}$ guesses)

Accordingly, the time complexity is $2^{119}$ encryptions.

The memory used for attack is occupied by the key table and the ciphertext table. The size of the key table, if indexed by the key values, is $2^{72}$ bits. The size of the ciphertext table is $2^{8}$ blocks ( 128 bits per block), if indexed by the plaintext values. Accordingly, the memory required for attack is about $2^{65}$ blocks.

\subsection{Key Recovery Attack on 12-Round CLEFIA}

We extend the impossible differential attack of the 11-round CLEFIA described in Sect. 3.2 by one round on the plaintext side. In addition to $R K_{22}, R K_{23}$, and $R K_{21,0}^{\prime}$, we can obtain the least significant byte of $R K_{0}$.

Movement of Whitening Key $\boldsymbol{W} \boldsymbol{K}_{\mathbf{0}}$. Move the whitening key $W K_{0}$, and place it at the bit-wise exclusive OR with the first round output $x^{\{1,0\}}$.

Plaintext Choice Method. Prepare a data set that comprises $2^{40}$ plaintexts in which the first three bytes of the first word, and the third and fourth words of the plaintext are fixed as shown in Fig. [5. In other words, there are $2^{40}$ plaintexts for which the first three bytes of the fourth word $x^{\{1,3\}}$, the second word $x^{\{1,1\}}$, and the third word $x^{\{1,2\}}$ are fixed, if taken as the first-round output. If, for each value of the first word $x^{\{1,0\}}$ of the first-round output, it is possible to choose $2^{8}$ plaintexts for which the least significant bytes of the fourth word $x^{\{1,3\}}$ are different (i.e., structures), the attack described in Sect. 3.2 can be applied.

Let the first word $x^{\{0,0\}}$ of the plaintext be $\left[a_{(8)}, b_{(8)}, c_{(8)}, d_{(8)}\right]$ and let $R K_{0}$ be $\left[k_{0(8)}, k_{1(8)}, k_{2(8)}, R K_{0,3(8)}\right]$. Here, $a, b$, and $c$ are arbitrary fixed values, and $d$ is a variable that takes values from 0 to 255 in order. Using this variable to express the first word $x^{\{1,0\}}$ of the first-round output, we get

$$
\begin{aligned}
x^{\{1,0\}} & =M_{0}{ }^{t}\left[S_{0}\left(a \oplus k_{0}\right), S_{1}\left(b \oplus k_{1}\right), S_{0}\left(c \oplus k_{2}\right), 0\right] \\
& \oplus M_{0}{ }^{t}\left[0,0,0, S_{1}\left(d \oplus R K_{0,3}\right)\right] \oplus x^{\{0,1\}} .
\end{aligned}
$$




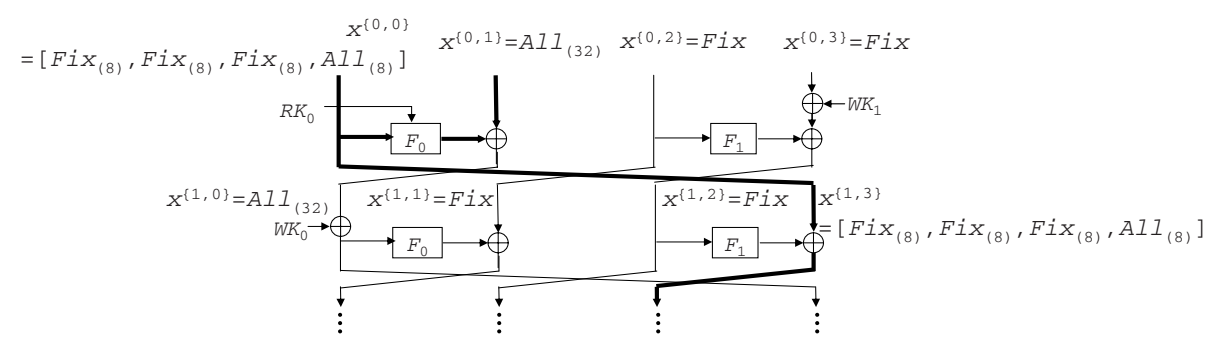

Fig. 5. Choice of plaintext for a one-round extension

The first term on the right side of (7) is a fixed value.

To choose $2^{8}$ plaintexts (structure) such that the least significant bytes of $x^{\{1,3\}}$ are all different for each value of $x^{\{1,0\}}$, we guess $R K_{0,3}$ and choose the data for which $x^{\{0,1\}}$ is $x^{\{1,0\}} \oplus M_{0}{ }^{t}\left[0,0,0, S_{1}\left(d \oplus R K_{0,3}\right)\right]$ corresponding to the change in $d$. Here, $x^{\{1,0\}}$ is actually the unknown value $x^{\{1,0\}} \oplus M_{0}{ }^{t}\left[S_{0}(a \oplus\right.$ $\left.\left.k_{0}\right), S_{1}\left(b \oplus k_{1}\right), S_{0}\left(c \oplus k_{2}\right), 0\right]$, but when choosing a single structure, we can fix the value of $x^{\{1,0\}}$. As a result, $2^{32}$ structures can be chosen for the first-round output.

Key Recovery. Because an attack in the same way as described in Sect. 3.2 is possible, this description follows the procedure of that section.

From among the ciphertext pairs that correspond to the plaintext pairs for which the second-round input difference is $[0,0,0,[0,0,0, X]]$, choose those for which the ciphertext difference is $[0,[Y, 0,0,0], \beta, \gamma]$. The probability of obtaining such ciphertext pairs is $2^{-80}$.

For the chosen ciphertext pair, the keys for which the 10th-round output difference $\left[\Delta x^{\{10,0\}}, \Delta x^{\{10,1\}}, \Delta x^{\{10,2\}}, \Delta x^{\{10,3\}}\right]$ is $[0,0,[Y, 0,0,0], 0]$ are wrong keys. Prepare a key table to distinguishing whether the $R K_{21,0}^{\prime}\left|R K_{22}\right| R K_{23}$ candidate is correct or wrong for each first-round guessed key $R K_{0,3}$. Keys obtained by differential table look-up with the input differences and the output differences for the 11 th-round $F_{1}$ and the 12 th-round $F_{1}$ are wrong keys. The probability of a wrong key obtained as an $R K_{21,0}^{\prime}\left|R K_{22}\right| R K_{23}$ candidate using the two differential tables is $2^{-40}$. Accordingly, the number of ciphertext pairs needed to narrow the 8-bit keys $R K_{0,3}$ and 72-bit keys $R K_{21,0}^{\prime}\left|R K_{22}\right| R K_{23}$ down to the correct key, $N$, is $2^{45.8}$ according to

$$
2^{80}\left(1-2^{-40}\right)^{N}=1 \text {. }
$$

When key $R K_{0,3}$ is wrong, all of the keys are wrong.

From the above description, $2^{45.8} / 2^{-80}=2^{125.8}$ plaintext pairs are required for attack. Here, by changing the order of choosing the plaintext-ciphertext pairs according to the guessing of key $R K_{0,3}$, the number of chosen plaintexts does not increase when guessing key $R K_{0,3}$. If we choose two different plaintexts from a single structure seen in the first-round output, we can make ${ }_{2} C_{2} \approx 2^{14.9}$ 


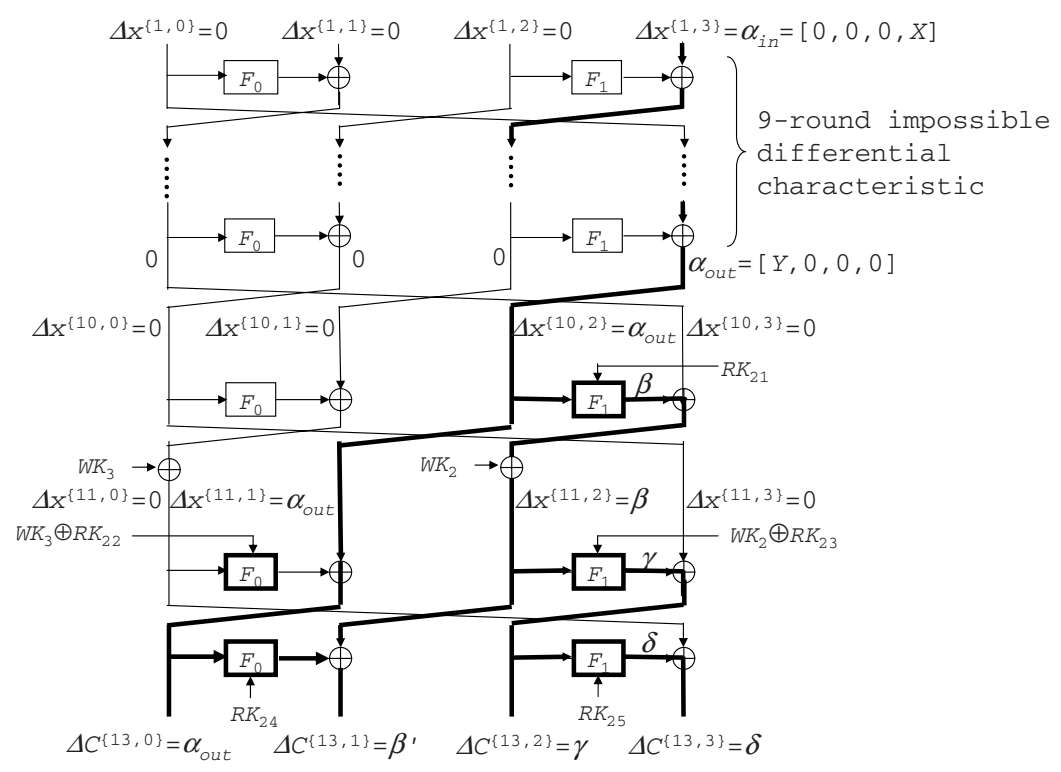

Fig. 6. Impossible differential attack on 13-round CLEFIA

pairs for which the difference is $[0,0,0,[0,0,0, X]]$. That is to say, if we prepare $2^{78.9}\left(=2^{125.8-32-14.9}\right)$ sets of $2^{40}$ plaintexts $\left(2^{32}\right.$ structures $)$ for which the first three bytes of the first word and the third and fourth words of the plaintext are fixed, we can obtain the number of ciphertext pairs required for attack. The number of plaintexts in that case is $2^{78.9} \cdot 2^{40}=2^{118.9}$. The difference in the required number of plaintexts with Sect. $3.2\left(2^{118.8}\right)$ arises from the difference in the number of ciphertext pairs $N$ required to narrow down the keys to the one correct remaining key using the key table.

The time complexity required for attack is as follows.

1. For obtaining the ciphertexts : $2^{119}$ encryptions

2. For reducing the key candidates : $2^{8} \cdot 2^{46} \cdot 2^{32}=2^{86}$ F-function computations $<2^{82}$ encryptions

(In detail, $R K_{0,3}$ guesses $2^{8} \cdot$ ciphertext pairs $2^{45.8} \cdot R K_{22}$ guesses $2^{32}$ )

Accordingly, the time complexity is $2^{119}$ encryptions.

The memory used for attack is occupied by the key table and the ciphertext table. The key table size is $2^{80}$ bits and the ciphertext table size is $2^{40}$ blocks. Accordingly, the memory size required for attack is about $2^{73}$ blocks.

\subsection{Key Recovery Attacks on 13 and 14-Round CLEFIA}

We present a 13-round CLEFIA attack for the key length of 192 bits or more shown in Fig. 6] and a 14-round CLEFIA attack for the key length of 256 bits.

In the 13-round attack, it is possible to obtain $R K_{0,3}$, the most significant byte of $R K_{21}$ (denoted as $R K_{21,0(8)}$ ), WK $K_{3} \oplus R K_{22}, W K_{2} \oplus R K_{23}, R K_{24}$ and 
$R K_{25}$. In the 14-round attack, it is possible to obtain $R K_{0,3}$, the most significant byte of $W K_{3} \oplus R K_{21}, R K_{22}, R K_{23}, W K_{3} \oplus R K_{24}$ and $W K_{2} \oplus R K_{25}, R K_{26}$, and $R K_{27}$. In the same way as done in Sects. 3.2 and 3.3. we first present the attack procedure for the 12 rounds from the second round to the 13 th round. Then, we extend one round on the plaintext side. Finally, we explain the 14-round attack.

Movement of Whitening keys $\boldsymbol{W} \boldsymbol{K}_{\mathbf{0}}, \boldsymbol{W} \boldsymbol{K}_{\mathbf{2}}$, and $\boldsymbol{W} \boldsymbol{K}_{\mathbf{3}}$. The whitening keys $W K_{0}, W K_{2}, W K_{3}$ are moved in the same way as in Sects. 3.2 and 3.3 .

Key Recovery on 12-Round CLEFIA. We choose the ciphertext pairs for which the first round output difference is $[0,0,0,[0,0,0, X]]$ and the 12 th-round output difference is $[[Y, 0,0,0], \beta, \gamma, 0]$ for use in attack. Here, $\beta$ represents the 255 values that can be obtained as the output difference when the input difference for $M_{1}$ is $[Y, 0,0,0] ; \gamma$ is a non-zero value.

From among the ciphertext pairs that correspond to the plaintext pairs for which the first round output difference is $[0,0,0,[0,0,0, X]]$, select those for which the differences are $\left[[Y, 0,0,0], \beta_{(32)}^{\prime}, \gamma, \delta_{(32)}\right]$. Here, $\beta^{\prime}$ is the bit-wise exclusive OR of the 255 values that $\beta$ can take with the 255 values that the $M_{0}$ output difference can take for the case in which the input difference of $M_{0}$ is $[Y, 0,0,0]$, or $255 \cdot 255 \approx 2^{16}$. The $\gamma$ and $\delta$ are non-zero values. The probability of obtaining such ciphertext pairs is

$$
255 / 2^{32} \cdot(255 \cdot 255) / 2^{32} \cdot\left(2^{32}-1\right) / 2^{32} \cdot\left(2^{32}-1\right) / 2^{32} \approx 2^{-40} .
$$

From among the chosen ciphertext pairs, classify the ciphertext pairs for which the 12th-round output difference is $[[Y, 0,0,0], \beta, \gamma, 0]$ by guessing the most significant byte of $R K_{24}$. Among the ciphertext pairs for which the difference is $\left[[Y, 0,0,0], \beta^{\prime}, \gamma, \delta\right]$, the probability that a usable ciphertext pair exists for each value of the most significant byte $R K_{24}$ is $2^{-8}$.

The keys for which the 10th-round output difference is $[0,0,[Y, 0,0,0], 0]$ are wrong keys. Prepare a table (key table) for distinguishing $R K_{21,0} \mid\left(W K_{2} \oplus\right.$ $\left.R K_{23}\right) \mid R K_{25}$ candidates as correct or wrong. Then, use the input differences and output differences for the 11 th-round and 12 th-round $F_{1}$ s and the 13 th-round $F_{1}$ for look-up in the differential table and mark the obtained keys as wrong. Here, to calculate the input values of the 11 th-round $F_{1}$ and the 12 th-round $F_{1}$, we guess the least significant three bytes of $R K_{24}$ and all of $W K_{3} \oplus R K_{22}$. The input of the 12 th-round $F_{0}$ can be calculated using the $R K_{25}$ candidates.

The probability of knowing that a $R K_{21,0}\left|\left(W K_{2} \oplus R K_{23}\right)\right| R K_{25}$ candidate is wrong by using the differential table for the three $F_{1} \mathrm{~s}$ is $2^{-72}$, from the average of $2^{-8}$ for the 11 th-round $F_{1}$ and the average of $2^{-32}$ for the 12 th-round and 13 thround $F_{1}$. Accordingly, the number of ciphertext pairs, $N$, required to narrow the 72-bit key $R K_{21,0}\left|\left(W K_{2} \oplus R K_{23}\right)\right| R K_{25}$ and 64-bit key $R K_{24} \mid\left(W K_{3} \oplus R K_{22}\right)$ down to the correct key is about $2^{78.6}$ from

$$
2^{136}\left(1-2^{-72}\right)^{N}=1
$$

From the above description, the number of plaintext pairs required for attack is $2^{78.6-40-8}=2^{126.6}$. 
If we choose two plaintexts from the same structure, we can make ${ }_{2} C_{2} \approx 2^{14.9}$ pairs for which the difference is $[0,0,0,[0,0,0, X]]$. That is to say, if we choose $2^{111.7}\left(=2^{126.6-14.9}\right)$ structures, we can obtain the number of ciphertext pairs required for attack. In that case, the number of plaintexts is $2^{111.7} \cdot 2^{8}=2^{119.7}$.

Key Recovery on 13-Round CLEFIA. We extend the method for attack the 12-round CLEFIA that is described above by one round on the plaintext side to break 13-round CLEFIA.

The number of ciphertext pairs, $N$, required to narrow down the 8-bit key $R K_{0,3}$, the 72-bit key $R K_{21,0}\left|\left(W K_{2} \oplus R K_{23}\right)\right| R K_{25}$ and the 64-bit key $R K_{24} \mid$ $\left(W K_{3} \oplus R K_{22}\right)$ to the one correct key using the key table is $2^{78.7}$ according to

$$
2^{144}\left(1-2^{-72}\right)^{N}=1 \text {. }
$$

The method for choosing structures for each value of the first word $x^{\{1,0\}}$ of the first round output is the same as described in Sect. 3.3. so the number of chosen plaintexts on the plaintext side is extended by $N$. Accordingly, the number of plaintexts required is $2^{119.8}$.

Prepare $2^{79.8}$ sets of $2^{40}$ plaintexts for which the first three bytes of the first word and the third and fourth words are fixed ( $2^{119.8}$ plaintexts in total). Regarding these plaintexts at the first round output, we can consider them to be $2^{79.8}$ sets of $2^{40}$ plaintexts with the first three bytes of the fourth word and second and third words fixed. We save these $2^{119.8}$ plaintexts in a table, guess $R K_{0,3}$, and choose the plaintext pairs and use them in attack. The reason for saving all of the data, which differs from the procedure of Sect. 3.3. is that there are more keys to be guessed on the ciphertext side, and it is not possible to have a key table for them 3

The time complexity required for attack is as follows.

1. For obtaining the ciphertexts : $2^{119.8}$ encryptions

2. For reducing the key candidates $: 2^{8} \cdot 2^{78.7} \cdot 2^{64}=2^{150.7}$ F-function computations $<2^{146}$ encryptions

(In detail, $2^{8} R K_{0,3}$ guesses $\cdot 2^{78.7}$ ciphertext pairs $\cdot 2^{64} W K 3 \oplus R K_{22}$ and $R K_{24}$ guesses)

Accordingly, the time complexity is $2^{146}$ encryptions.

The memory used for attack is occupied by the key table and the ciphertext table. The size of the key table is $2^{72}$ bits; the size of the ciphertext table is $2^{119.8}$ blocks. Accordingly, the memory required for attack is about $2^{120}$ blocks.

Key Recovery on 14-Round CLEFIA. 14-round CLEFIA can be broken by adding exhaustive search of the 14 th-round keys $R K_{26}$ and $R K_{27}$ to the 13 -round attack. The number of chosen plaintexts required for attack is $2^{120.3}$, because the number of ciphertext pairs, $N$, required for narrowing the keys down to the correct key using the key table is about $2^{79.2}$, from

$$
2^{208}\left(1-2^{-72}\right)^{N}=1 .
$$

3 In this paper, it is not possible to have a table that exceeds $2^{128}$ blocks. 
The time complexity is as follows.

1. For obtaining the ciphertexts : $2^{120.3}$ encryptions

2. For reducing the key candidates : $2^{8} \cdot 2^{79.2} \cdot 2 \cdot 2^{128}=2^{216.2}$ F-function computations $<2^{212}$ encryptions

(In detail, $2^{8} R K_{0,3}$ guesses $\cdot 2^{79.2}$ ciphertext pairs $\cdot 2^{128}$ guesses for $R K_{22}$, $W K 3 \oplus R K_{26}$ and $R K_{27}$ guesses)

Accordingly, the time complexity is $2^{212}$ encryptions.

The memory used for attack is occupied by the key table and the ciphertext table. The size of the key table is $2^{72}$ bits; the size of the ciphertext table is $2^{120.3}$ blocks. Accordingly, the amount of memory required for attack is about $2^{121}$ blocks.

\section{Conclusion}

We have presented previously unknown 9-round impossible differentials in CLEFIA, which are impossible differentials that exist in structures designed by using DSM. We used these impossible differentials to apply impossible differential attacks on CLEFIA. The result showed that an impossible differential attack that is more efficient than exhaustive search is possible for 128-bit key, 12-round CLEFIA. Furthermore, attack of 13-round CLEFIA and 14-round CLEFIA is possible for key lengths of 192 bits and 256 bits, respectively. The number of chosen plaintexts, the time complexity, and the amount of memory required for attack are listed in Table 2 .

Table 2. Results of impossible differential attacks

\begin{tabular}{|c|c|r|c|c|c|}
\hline Reference & $\begin{array}{c}\text { Number of } \\
\text { rounds }\end{array}$ & Key length & $\begin{array}{c}\text { Chosen } \\
\text { plaintexts }\end{array}$ & $\begin{array}{c}\text { Time complexity } \\
\text { (encryptions) }\end{array}$ & $\begin{array}{c}\text { Amount of memory } \\
\text { (blocks) }\end{array}$ \\
\hline$[6] 7$ & 10 & $128,192,256$ & $2^{101.7}$ & $2^{102}$ & $2^{32}$ \\
\hline$[6]$ & 11 & 192,256 & $2^{103.5}$ & $2^{188}$ & $2^{121}$ \\
\hline$[6]$ & $12^{*}$ & 256 & $2^{103.8}$ & $2^{252}$ & $2^{73}$ \\
\hline This paper & 12 & $128,192,256$ & $2^{118.9}$ & $2^{119}$ & $2^{120}$ \\
\hline This paper & 13 & 192,256 & $2^{119.8}$ & $2^{146}$ & $2^{121}$ \\
\hline This paper & 14 & 256 & $2^{120.3}$ & $2^{212}$ & \\
\hline
\end{tabular}

* Without whitening key

Even though the 9-round impossible differentials presented in this paper have the same number of rounds characteristic as the impossible differentials identified by the designers, our impossible differential attacks exceed the designers' evaluation by two more rounds that can be broken for each key length. That is true for the following reason. For the impossible differentials found by the designers, the length of the parts of the plaintext differences and ciphertext differences that are not zero is 32 bits, and the plaintext differences and ciphertext differences must be the same. For our impossible differentials, however, the length of 
those parts is 8 bits, and it is not necessary for the plaintext differences and the ciphertext differences to be the same, that is, they are truncated differences. If the number of bits for which the difference is non-zero is small, the number of round key bits related to the difference is also small. Because it is possible to obtain round keys that span many rounds, the number of rounds that can be broken can be increased. Also, because it is a truncated difference, the probability of obtaining ciphertext that can be used in attack is high, and we were able to increase the number of rounds that can be broken by reducing the number of chosen plaintexts that are required. Other reasons for the successful attack are the movement of the whitening key and the use of the differential table method that is often used in differential attacks. Because the number of CLEFIA rounds is 18 for a key length of 128 bits, 22 for a 192-bit key and 26 for a 256-bit key, the impossible differential attacks presented in this paper do not affect the security of CLEFIA. These attacks can, however, break more rounds of than other CLEFIA attack methods.

There is currently no method for guaranteeing resistance to an impossible differential attack and no method for designing a block cipher that is resistant to an impossible differential attack. Accordingly, much time should be allocated to evaluation of block cipher with respect to impossible differential attacks. Furthermore, methods for guaranteeing resistance to an impossible differential attack and methods for designing block ciphers that resist impossible differential attacks are important topics for future research.

Acknowledgments. The authors would like to thank Takeshi Kawabata, Hiroki Nakashima, Takahiko Syouji, and Akira Nozawa for their helpful comments.

\section{References}

1. Biham, E., Biryukov, A., Shamir, A.: Cryptanalysis of Skipjack Reduced to 31 Rounds Using Impossible Differentials. In: Stern, J. (ed.) EUROCRYPT 1999. LNCS, vol. 1592, pp. 12-23. Springer, Heidelberg (1999)

2. Biham, E., Shamir, A.: Differential Cryptanalysis of DES-like Cryptosystems. In: Menezes, A. J., Vanstone, S.A. (eds.) CRYPTO 1990. LNCS, vol. 537, pp. 2-21. Springer, Heidelberg (1991)

3. Matsui, M.: Linear Cryptanalysis Method for DES Cipher. In: Helleseth, T. (ed.) EUROCRYPT 1993. LNCS, vol. 765, pp. 386-397. Springer, Heidelberg (1994)

4. Shirai, T., Preneel, B.: On Feistel Ciphers Using Optimal Diffusion Mappings Across Multiple Rounds. In: Lee, P.J. (ed.) ASIACRYPT 2004. LNCS, vol. 3329, pp. 1-15. Springer, Heidelberg (2004)

5. Shirai, T., Shibutani, K.: On Feistel Structures Using a Diffusion Switching Mechanism. In: Robshaw, M.J.B. (ed.) FSE 2006. LNCS, vol. 4047, pp. 41-56. Springer, Heidelberg (2006)

6. Shirai, T., Shibutani, K., Akishita, T., Moriai, S., Iwata, T.: The 128-bit Blockcipher CLEFIA (Extended Abstract). In: Biryukov, A. (ed.) FSE 2007. LNCS, vol. 4593, pp. 181-195. Springer, Heidelberg (2007)

7. Sony Corporation. The 128-bit Blockcipher CLEFIA, Security and Performance Evaluations, Revision 1.0, June 1 (2007), http://www.sony.co.jp/Products/clefia/ 